\title{
Research brief
}

\section{Receptor modelling and risk assessment of volatile} organic compounds measured at a regional background site in South Africa North-West University's research published in Atmospheric Environment

\author{
Kerneels Jaars ${ }^{1}$, Mika Vestenius ${ }^{2}$, Pieter G. van Zyl ${ }^{1}$, Johan P. Beukes ${ }^{1}$, Heidi Hellén ${ }^{2}$, \\ Ville Vakkari ${ }^{2}$, Marcell Venter ${ }^{1}$, Miroslav Josipovic ${ }^{1}$ and Hannele Hakola ${ }^{2}$ \\ ${ }^{1}$ Chemical Resource Beneficiation, North-West University, Potchefstroom, South Africa \\ ${ }^{2}$ Finnish Meteorological Institute, Atmospheric Composition Unit, P.O. Box 503, 00101 Helsinki, Finland
}

\section{http://dx.doi.org/10.17159/2410-972X/2018/v28n2a9}

Volatile organic compounds (VOCs) can have significant impacts on climate and human health. In order to develop climate change reduction strategies and to assess the impacts of VOCs on human health, it is crucial to determine the sources of VOCs, which can be emitted from biogenic and anthropogenic sources. In this study source apportionment was performed using positive matrix factorisation (PMF) analysis on VOC data collected at Welgegund - a regional background station located on a commercial farm approximately $100 \mathrm{~km}$ west of Johannesburg that is influenced by the major sources in the interior of South Africa - for more than two years. In addition, a risk assessment study was also performed in view of the major source regions affecting Welgegund in order to quantify the impacts of anthropogenic VOCs measured at Welgegund on human health.

PMF analysis indicated ten meaningful factor solutions, with five factors being associated with biogenic emissions and five with anthropogenic sources. Three of the biogenic factors were characterised by a specific biogenic species, i.e. isoprene, limonene and 2-methyl-3-buten-2-ol (MBO), while the other two biogenic factors comprised mixtures of biogenic species with different tracer species. The temporal factor contribution for the isoprene, limonene and MBO factors correlated relatively well with the seasonal wet pattern. One anthropogenic factor was associated with emissions from the JohannesburgPretoria megacity, the Vaal Triangle and the Mpumalanga Highveld source regions, while another anthropogenic factor could be related to coal combustion. An anthropogenic factor was also identified that reflected the influence of solvents on atmospheric VOC concentrations, while two anthropogenic factors were determined that indicated the influence of farming activities in proximity to Welgegund.
A lifetime cancer risk (LCR) and non-cancer hazard ratio (HR) assessment study conducted for VOCs in relation to three source regions indicated that the non-cancerous influence of VOCs measured in the source regions is significantly lower compared to the cancerous influence of these species on human health, which raises concern. However, LCR values were within an acceptable range. Factor analysis also identified sources that could be targeted to minimise VOC-related LCRs and HRs, which included benzene-related cancers that can be reduced by targeting incomplete combustion sources and coal combustion. 